Chapter 20

\title{
Pharmacological Treatments for Type 2 Diabetes
}

\author{
Roberto Pontarolo, \\ Andréia Cristina Conegero Sanches, Astrid Wiens, \\ Helena Hiemisch Lobo Borba, Luana Lenzi and \\ Suelem Tavares da Silva Penteado
}

Additional information is available at the end of the chapter

http://dx.doi.org/10.5772/56456

\section{Introduction}

Type 2 diabetes mellitus (T2DM) results from relative defects in insulin secretion and action. T2DM may be associated with metabolic syndrome, and it is characterized by insulin resistance, android obesity, dyslipidemia and hypertension. Furthermore, it is responsible for increased morbidity and mortality related to cardiovascular diseases.

In Latin America, diabetes is a major health problem, where the prevalence has reached more than 19 million people [1], many of whom are at the productive age for work. This phenomenon has resulted in an increased burden on social security, thereby fueling the continuation of the vicious cycle of poverty and social exclusion.

In terms of morbidity, diabetes mellitus currently represents one of the major chronic diseases affecting people today, including individuals in countries at all stages of economic and social development. Even developed countries, which, despite scientific advances and easy access to health care, are affected by the increasing prevalence of diabetes. Thus, it is assumed that interventions aimed at preventing this disease, such as physical activity and diet, are underutilized [2].

The relevance of diabetes has increased in recent decades as a result of various factors, such as a high urbanization rate, increased life expectancy, industrialization, hypercaloric diets rich in carbohydrates, displacement of populations to urban areas, changes in lifestyle, physical inactivity and obesity. It is estimated that by 2020, two-thirds of the disease burden will be attributed to chronic, noncommunicable diseases [3]. High-calorie diets and sedentary 
lifestyles are the major factors contributing to the increased prevalence of obesity, representing additional major risk factors for T2DM [4].

Diabetes is the seventh leading cause of death in the United States. Among adults diagnosed with either type 1 or type 2 diabetes, 12 percent take insulin only, 14 percent take both insulin and oral medication, 58 percent take oral medication only, and 16 percent do not take either insulin or oral medication [5].

The treatment of diabetes is aimed predominantly at glycemic control. The treatment objectives are to relieve the symptoms, improve the quality of life, prevent acute and chronic complications, reduce mortality and treat the disease. The basic strategies for treatment and disease control of type 1 and 2 diabetes consist primarily of a specific diet, physical activity and the appropriate use of medication (oral agents and / or insulin). The outcome of the treatment depends on providing diabetic patients with education and ensuring that they adopt specific behavioral measures and practices.

Currently, there is a tendency to use unconventional measures of care for patients with chronic diseases, such as methods involving nonpharmacological treatment [6], emphasizing continued practice and frequent daily exercise and walks. These approaches also emphasize proper nutrition, which is now regarded more as a benefit than as a punishment. The use of medications is indicated for T2DM, together with diet and increased physical activity $[7,8]$.

Because it is not always possible to establish a full behavioral change in the high-risk population, and even trying to improve the prevention of T2DM can be difficult, a number of drugs have been tested with the intention of preventing this disease. Several drugs have been used, and some of the key studies are summarized here, including drugs such as oral anti-diabetic agents and other oral medications.

In obese patients with T2DM, the priority is weight loss. If glycemic control is not achieved after 4 to 6 weeks, drugs that sensitize the action of insulin (biguanide and thiazolidinedione) may be indicated, either in combination or not with anti-obesity drugs. If satisfactory glycemic control is not achieved, drugs that reduce the intestinal absorption of glucose (acarbose or miglitol) or that enhance insulin secretion (sulfonylurea, repaglinide or netaglinida) may be used.

In type 2 diabetic normal weight or overweight (body mass index $<30 \mathrm{~kg} / \mathrm{m}^{2}$ ) individuals, sulfonylureas, repaglinide or nateglinide can be tried initially. If adequate glycemic control is not achieved after 2 to 4 weeks, biguanide, thiazolidinedione or an inhibitor of intestinal absorption of glucose can be added $[7,8]$.

Often, the effectiveness of the chosen pharmacological treatment cannot be predicted. Pharmacotherapeutic failure or undesired effects that lead to other health problems, such as adverse reactions and toxicities, may arise. These events are regarded as a negative outcomes associated with the medication use.

In some cases, negative results associated with the use of a medication are inevitable, as in the case of some adverse reactions. However, negative reactions can sometimes be avoided, such as those that result from the inappropriate use of a drug or problems with the monitoring of 
a drug's effects. The occurrence of these avoidable outcomes could be reduced by adequate follow-up of patients [9].

Non-effective treatment of T2DM leads to a significant increase in the values of glycated hemoglobin, which leads to a decreased quality of life for patients and a significant economic impact $[10,11]$. The consequences of diabetes on health systems reflect only a fraction of the damage that is caused to individuals, their families and society.

In recent years, great progress in the treatment of T2DM has been observed. Different classes of drugs are used for this purpose, including analog and human insulin, drugs that act by reducing insulin resistance (biguanides and thiazolidinediones), secretagogues and their analogs (sulfonylureas, metglinides, inhibitors of DPP-4 or GLP-1 agonists) and drugs that reduce the rate of degradation of carbohydrates (alfaglicosidase inhibitors). Some treatments have been used for several decades, such as human insulin, which was first isolated in the 1930s. Other drugs have been developed over the last century, including metformin, which is the most used oral antidiabetic in the U.S. today, and liraglutide, an analog of GLP-1.

The current treatments aim to reduce insulin resistance and maintain adequate glycemic control to prevent or reduce microvascular and macrovascular complications by improving the function of the pancreatic beta cells through interventions involving diet, exercise, oral hypoglycemic agents, anti-hyperglycemic agents and / or anti-obesity drugs. There are currently several types of treatments, which may be used alone or in combination.

Among the class of biguanides, which sensitize the action of insulin, are metformin and phenformin. Metformin is used more frequently than phenformin and has fewer side effects (diarrhea, metallic taste and nausea - which may decrease with continued use). Among the advantages of this class of drugs is its anorectic effect, which aids in weight loss, and the fact that it does not cause hypoglycemia (it does not stimulate insulin secretion).

In the United States and some European countries, other classes of antidiabetic thiazolidinedione derivatives are available, such as rosiglitazone and pioglitazone, which act by increasing the sensitivity of the liver, muscles and adipocytes to insulin, resulting in a decrease in peripheral resistance. The use of troglitazone, which belongs to this therapeutic class, was suspended due to hepatotoxicity. Rosiglitazone is more potent and has lower liver toxicity and fewer interactions with other drugs because it does not induce metabolism by cytochrome P450 (CYP) 3A4. The side effects include upper respiratory tract infections, headache, elevated transaminases, edema, weight gain and anemia. Pioglitazone is also associated with less liver toxicity and has the same mechanism of action and side effects as rosiglitazone. However, pioglitazone interacts with some medications.

The competitive alpha-glucosidase inhibitors, such as acarbose, miglitol and voglibose, act as antagonists of sucrase and amylase, and they also decrease the intestinal absorption of glucose. The most frequent side effects of alpha-glucosidase inhibitors are bloating, diarrhea, abdominal pain and elevated transaminases, and they are contraindicated in cases of inflammatory bowel disease, pregnancy and lactation, hepatic or renal impairment.

Another class of drugs used to treat T2DM is the class of sulfonylureas, including chlorpropamide (first generation), glibenclamide, gliclazide and glipizide (the second generation) and 
glimepiride (third generation). These drugs act as insulin secretagogues, and the side effects are hypoglycaemia, hematological (leukopenia, agranulocytosis, thrombocytopenia and hemolytic anemia) and gastrointestinal (nausea, vomiting, more rarely cholestatic jaundice) complications and allergic reactions. They may also cause an increase in weight as a result of binding to the plasma proteins, and their effects can be enhanced by the use of other drugs concomitantly, causing hypoglycemia.

Additional insulin secretagogues include repaglinide, nateglinide and meglitinida. Derivatives of benzoic acid and the amino acid D-phenylalanine increase insulin secretion through an action similar to that of the sulfonylureas. Due to their rapid absorption, this action begins approximately 30 minutes after administration. These drugs do not have interactions with other medications and are not contraindicated during pregnancy, lactation or in the presence of other pathologies.

In many diabetic patients, a hypocaloric diet aimed at weight reduction alone is able to control glucose levels. The anti-obesity effects of catecholaminergic (amfepramone, fenproporex, mazindol), serotonergic (fluoxetine, sertraline) and mixed action catecholaminergic and serotonergic (such as sibutramine) drugs that affect appetite and the induction of satiety may be used. In addition to these, orlistat or tetrahydrolipstatin, which inhibit intestinal lipase and fat absorption, may allow a reduction of the dose of hypoglycemic medications.

The use of insulin in the treatment of T2DM reverses diabetes symptoms and may be used in those with severe hyperglycemia with ketonemia or ketonuria, newly diagnosed patients, or in diabetics who do not respond to treatment with diet, exercise and / or treatments with oral hypoglycemic agents with anti-hyperglycemic or insulin-sensitizing action. Initially, human insulin in association with porcine insulin and regular or single insulin were used. There is a high chance of developing hypoglycemia as a result of the use of interprandial insulin analogous, which have faster action than human insulin. Currently, several types of insulin analogs have been synthesized: lispro, aspart and glargine. These analogs differ in their speed of action and duration of effect due to structural changes in the position of the amino acid chains of insulin. There are multiple options for the rout of administration, and they can be used in combination with oral hypoglycemic agents.

New drugs for the treatment of diabetes are emerging, making multiple therapeutic options possible. Furthermore, the use of insulin by inhalation has been studied to reduce the difficulties associated with subcutaneous administration.

The aim of this chapter was to evaluate all of the available treatments for T2DM, their mechanisms of action and side effects, and also to describe the emerging drugs and trends that are anticipated in the coming years. A better understanding of the mechanisms of action of drugs and the adverse reactions associated with them is important for health professionals and caregivers of patients with T2DM. 


\section{Treatments}

\subsection{Biguanides}

Metformin and phenformin are oral antidiabetic drugs of the biguanide class. Metformin is the drug of choice for treatment of adults with T2DM due to its low frequency of side effects. It is currently used by nearly one-third of diabetic patients in Italy and is prescribed in the U.S. (> $40 \mathrm{~m}$ million prescriptions in 2008). Phenformin is no longer marketed in many countries, although it is still available in Italy $[12,13]$.

Both metformin and phenformin facilitate weight loss in obese nondiabetic patients without appreciably reducing glucose levels in the blood of such individuals. This weight loss is attributed to the anorectic effect and the slight reduction in the gastrointestinal absorption of carbohydrates [14].

\subsubsection{Metformin}

Metformin is marketed in tablets of 500 or $850 \mathrm{mg}$, and the maximum dose is $2.5 \mathrm{~g} /$ day, although there are no reports in the literature on the use of metformin at doses up to $3 \mathrm{~g}$ when administered after meals to minimize gastrointestinal side effects [15]. It has been reported that this drug increases the number and improves the affinity of insulin receptors in adipocytes and muscle. In the muscle, metformin increases glucose uptake by 15 to $40 \%$ and stimulates glycogenolysis. In adipocytes, metformin inhibits lipolysis and the availability of free fatty acids (FFA). Moreover, metformin improves insulin action in the liver, decreasing hepatic production of glucose by 10 to $30 \%$ and, at the cellular level, it increases the tyrosine kinase activity of the insulin receptor, stimulating translocation of GLUT4 and the activity of glycogen synthase [16].

The use of metformin also improves the lipid profile, resulting in a decrease in the triglyceride levels by 20 to $25 \%$, a decrease in LDL-cholesterol by as much as $10 \%$, an increase in HDLcholesterol by $17 \%$, and a decrease in the level of factor plasminogen activator inhibitor (PAI-1) by $20-30 \%$. Insulin secretion in response to stimuli may remain unchanged or decrease as a result of the anorectic effect, which helps with weight loss. In addition to being associated with weight reduction, its effectiveness in glycemic control is similar to that of sulfonylurea [17]. Another advantage of metformin is that it does not induce hypoglycemia or stimulate insulin secretion [18].

The isolated use of metformin in T2DM lowers blood glucose by approximately $25 \%$, or 60 to $70 \mathrm{mg} / \mathrm{dl}$, and glycosylated hemoglobin is reduced by 1.5 to $2 \%$ [16]. The intensive glucose control resulting from the use of metformin significantly decreases the risk of cardiovascular disease and mortality related to diabetes and patients presented less weight gain compared to other medications including insulin. Metformin also avoids the inconvenience of hypoglycemia induced by treatment with insulin or sulfonylureas [19].

Metformin is absorbed in the intestine and excreted by the kidneys. It is minimally metabolized by the liver, has a low affinity for mitochondrial membranes, and it does not interfere with 
oxidative phosphorylation. Metformin is indicated as a monotherapy in obese diabetic or glucose intolerant patients. Approximately 5 to $10 \%$ of patients each year will not have an appropriate response to the drug. In these cases, to achieve satisfactory control, metformin can be used in combination with sulfonylureas, acarbose, thiazolidinedione, repaglinide and / or insulin [7, 20-24].

The most frequent side effects are diarrhea (15\%), a metallic taste and nausea, and these often decrease with continued use of the medication. The occurrence of lactic acidosis is rare $(0.03$ to 0.4 / 1000/year), occurring most often in people who have a contraindication to metformin, such as chronic liver disease (elevated transaminase 2 to 3 times normal values), heart failure, respiratory or renal failure (creatinine clearance $<70 \mathrm{ml} / \mathrm{min}$ or serum creatinine $\geq 1.5 \mathrm{mg}$ / dl). Metformin is not advisable for use in people over age 80, pregnant women, infants or alcoholics. In patients with proteinuria or those who are subjected to radiological examination containing iodine, it is prudent to provide adequate hydration and discontinue the medication a few days before [18]. Metformin shows synergistic effects with cimetidine and may decrease the absorption of vitamin B12 [15].

\subsubsection{Phenformin}

Phenformin presents a hypoglycemic action based on its insulin-sensitizing properties, similar to metformin. Approximately $30 \%$ of phenformin has hepatic metabolism and shows a high affinity for mitochondrial membranes, and it may also interfere with oxidative phosphorylation [18].

Due to its greater propensity to cause serious and fatal adverse events such as lactic acidosis, this drug was withdrawn from clinical practice in the 1970s. These effects are attributed to inhibition of Complex I of the mitochondrial respiratory chain. However, though no longer in clinical use, phenformin remains a widely used research tool to help delineate the cellular and molecular mechanisms that underlie the action of biguanides [25].

\subsection{Thiazolidinediones}

The thiazolidinediones (TZD) are popularly known as glitazones. Among the representatives of TZD are troglitazone (withdrawn from the market due to liver toxicity), rosiglitazone and pioglitazone (second generation thiazolidinediones).

Widely used in the treatment of T2DM, these drugs exert their effect by increasing and sensitizing insulin action in the liver, muscle and fat cells, decreasing peripheral resistance. They activate the intracellular nuclear receptors (PPAR-gamma, peroxisome proliferator activated receptor), which regulate the expression of genes involved in the metabolism of glucose and lipids, genes that are responsible for glucose uptake mediated by insulin in the peripheral tissues and genes that participated in the differentiation of preadipocytes into adipocytes. Moreover, these drugs inhibit peripheral lipolysis in adipocytes and assist in reducing the levels of free fatty acids and visceral adiposity, resulting in improvement of glycemic and metabolic parameters in these patients. They show good results in maintaining 
long-term glycemic control compared with other therapeutic options such as sulfonylureas and metformin [26-28].

Thiazolidinediones decrease glucose levels by approximately $20 \%$, but no increase insulin secretion is observed. These drugs inhibit oxidation of long chain fatty acids in the liver, and they decrease gluconeogenesis and the availability of free fatty acids. Although they induce a decrease of triglycerides by 15 to $20 \%$ and an increase of HDL-cholesterol of 5 to $10 \%$, the total cholesterol and LDL-cholesterol levels may not change or they may increase by 10 to $15 \%$ [18]. When compared to metformin, it has been observed that troglitazone has a greater potentiating effect for peripheral insulin action and less of an effect for reducing the hepatic glucose output. The association of thiazolidinedione with metformin is interesting because these drugs have additive effects [22].

Thiazolidinediones also increase the expression of the glucose transporter (GLUT4) and lipoprotein lipase, and they reduce the expression of leptin and tumor necrosis factor (TNFalpha). These results make this drug class the most widely prescribed for the treatment of T2DM [18, 29].

Side effects occur in less than $5 \%$ of patients, consisting of upper respiratory tract infections, headache, elevated transaminases, edema, weight gain and anemia. Hypoglycemia can occur when the use of thiazolidinediones is concomitant with secretagogues or insulin. Their use is contraindicated in children, pregnant women, or in individuals with elevated transaminase levels that are 2-3 times the values of reference [18].

\subsubsection{Troglitazone}

In mice subjected to arterial injury, troglitazone inhibited the growth of vascular smooth muscle cells and intimal hyperplasia, suggesting that thiazolidinediones decrease the progression of atherosclerosis. In diabetic patients treated with troglitazone, decreases were observed in platelet aggregation, factor plasminogen activator inhibitor (PAI-1) and blood pressure levels. These multiple effects strengthen its indication for the treatment of metabolic syndrome. However, caution is recommended with the indication because of the possibility of hepatic complications, including reports of fatalities associated with the use of troglitazone. Additionally, this drug should be administered with caution in cardiac patients due to the possibility of edema $[18,30,31]$.

\subsubsection{Pioglitazone}

Pioglitazone can be used as monotherapy or in combination with metformin, which has antihyperglycemic effects, or with sulfonylurea, meglitinida, or even insulin, especially in diabetic patients with metabolic syndrome. The dose varies from 15 to $45 \mathrm{mg}$, and it may be administered once a day. This drug displays mechanism of action and similar side effects to rosiglitazone, and it causes less liver toxicity than troglitazone. However, pioglitazone may interact with other drugs metabolized by P450 enzymes through changing their serum levels. An example is a decrease by approximately $30 \%$ of the contraceptive effect of ethinyl estradiol and norethindrone. Accordingly, the dose of the contraceptive must be increased in diabetic 
women who do not wish to become pregnant. The pharmacokinetics of pioglitazone are not altered by mild to moderate renal insufficiency [32].

\subsubsection{Rosiglitazone}

Rosiglitazone is more potent and has lower hepatotoxicity than troglitazone. Furthermore, it stimulates metabolism by cytochrome P450 (CYP) 3A4 without interacting with oral contraceptives, digoxin, ranitidine or nifedipine. Rosiglitazone dosage varies from 4 to $8 \mathrm{mg}$, and it may be given once a day. Similar to pioglitazone, rosiglitazone's pharmacokinetics are not altered by mild to moderate renal insufficiency, and dose modification is not required [33].

Recently published safety data prompted concerns about a possible association between the chronic use of rosiglitazone and an increased risk of cardiovascular events, leading to some parsimony in the use of TZDs in clinical practice. Furthermore, studies have recently shown bone loss and increased fracture among users of these medications in [27, 29, 34].

\subsection{Alpha-glucosidase inhibitors}

The competitive inhibitors of alpha-glucosidase, such as acarbose, miglitol and voglibose, act as enzymatic antagonists of oligosaccharide (e.g. amylase, maltase and sucrase) and decrease the intestinal absorption of glucose, particularly postprandial absorption, thereby modulating the insulin secretion [35]. These inhibitors present the advantage of lowering the incidence of cardiovascular events, and they have no systemic absorption [36].

More specifically, alpha-glucosidase is inhibited competitively, and its availability for oligosaccharides derived from the diet is reduced. Thus, the formation of monosaccharide decreases, and less insulin is required for metabolism, which leads to a reduction of glucose (because it is not absorbed) as well as postprandial insulin-induced increases [37]. These effects reflect a significant decrease in glycated hemoglobin [38, 39], which is more evident in highly hyperglycemic patients. Hyperglycemia in patients with mild or moderate glycemic control is less common than in those using other oral antidiabetic agents. In such cases, competitive inhibitors of alpha-glucosidase can be used in combination with insulin or any other oral hypoglycemic agents.

\subsubsection{Acarbose}

Acarbose has microbial origin and is structurally similar to natural oligosaccharides, having an affinity 104-105 times higher than drugs of the same class of alpha-glucosidases. With regard to the pharmacokinetic aspects, acarbose is poorly absorbed in the intestine (less than $2 \%$ ). The products produced by bacterial enzymes cleave acarbose, yielding intermediate 4-metipirogalol, which is conjugated and excreted as sulfates or glucuronidate [39].

In a prospective, randomized, double-blind, placebo-controlled trial [40], there was a satisfactory control of fasting and postprandial glucose with acarbose in T2DM. In a multicenter, randomized, double-blind, placebo-controlled clinical trial [41] conducted in patients with T2DM who were subjected to a specific diet and use of insulin, the patients showed decreased 
levels of blood glucose and glycated hemoglobin as well as a reduced daily requirement for insulin.

In a systematic review [42], it was concluded that acarbose inhibits postprandial hyperglycemia by lowering insulin levels after a glucose overload. However, it presents no advantages with respect to corporal weight or lipid metabolism, and there are no statistically significant effects on mortality, morbidity and quality of life in patients with T2DM. Compared with placebo, acarbose reduces $\mathrm{HbA1c}$ and fasting plasma glucose and postprandial glucose. Compared with sulfonylureas, it reduces glycemic control and has major adverse effects, particularly gastrointestinal.

\subsubsection{Voglibose}

Voglibose also has a microbial origin, and only 3-5\% of the drug is absorbed at the intestinal level. It is a potent inhibitor of alpha-glucosidase, but it is weaker than acarbose in the inhibition of sucrase and has little effect on pancreatic alpha-amylase [39].

\subsubsection{Miglitol}

Miglitol has a synthetic origin. It is absorbed rapidly through a transport mechanism in the jejunum that is partly identical to glucose, and it is quantitatively excreted unchanged by the kidney. Miglitol differs from acarbose, as it does not inhibit alpha-amylase, but rather it inhibits intestinal isomaltase [39].

The most frequent side effects of alpha-glucosidase inhibitors are seen at the intestinal level: flatulence, diarrhea, abdominal pain and elevated transaminases. The occurrence of hypoglycemia and an increase in body weight are rare because the agent does not stimulate insulin release or hypersecretion. These effects are only observed when miglitol is combined with other therapies. Its use is contraindicated in cases of inflammatory bowel disease, pregnancy and lactation, and hepatic or renal impairment.

\subsection{Sulfonylureas}

Another class of drugs used in the treatment of T2DM is the class of sulfonylureas, chlorpropamide and tolbultamide (first generation), glibenclamide, glipizide and gliclazide (second generation) and glimepiride (third generation). This class was introduced commercially in the 1950s and has since been recognized as first-line therapy and as a monotherapy or in combination [43]. The sulfonylureas are the drugs of choice for type 2 diabetics who do not benefit exclusively from diet and exercise [44].

These drugs act as insulin secretagogues and exert their main action on islet B cells, stimulating insulin secretion and thereby reducing the plasma glucose concentration [45]. The mechanism of action involves binding of the drug to a subunit of the ATP-sensitive potassium channels in the plasma membranes of B cells. The channels are closed, leading to a change in the membrane voltage, calcium influx and exocytosis of insulin granules [46, 47]. 
The basal secretion and insulin secretory response to various stimuli are intensified in the early days of treatment with sulfonylureas. With longer-term treatment, insulin secretion continues to increase, and tissue sensitivity to insulin also improves by an unknown mechanism [45].

The sulphonylureas are well absorbed after oral administration, and most reach peak plasma concentrations in 2-4 hours. The duration of the effect varies. All of these drugs bind tightly to plasma albumin and are involved in interactions with other drugs (e.g., salicylates and sulfonamides) such that there is competition for binding sites. The sulfonylureas (or their active metabolites) are mostly excreted in the urine; thus, their action is increased in elderly patients or those with renal disease [45].

\subsubsection{First-generation sulfonylureas}

The action of chlorpropamide and tolbultamide is long lasting, with substantial excretion in urine. Therefore, these drugs can cause severe hypoglycemia in elderly patients where there is a progressive decline in glomerular filtration. They cause flushing after alcohol consumption and exert similar effects to the diuretic hormone on the distal nephron, producing hyponatremia and water intoxication [45].

\subsubsection{Second-generation sulfonylureas}

The second-generation sulfonylureas (glibenclamide, glipizide and gliclazide) are more potent, but their hypoglycemic effects are not much greater, and failure to control blood glucose is as commonly observed as that with tolbutamide. All of these drugs contain the sulfonylurea molecule, but different substitutions result in pharmacokinetic differences, and thus differences in the duration of action. Glibenclamide should be avoided in the elderly and in patients with mild renal impairment because of the risk of hypoglycemia, as several of its metabolites are excreted in the urine and are moderately active [45].

The sulfonylureas cross the placenta and stimulate insulin release by fetal B cells, causing severe hypoglycemia at birth. Consequently, their use is contraindicated during pregnancy, and gestational diabetes is treated by diet supplemented where necessary with insulin [45].

In general, sulfonylureas are well tolerated. The observed side effects are hypoglycemia, hematological (leukopenia, agranulocytosis, thrombocytopenia, and hemolytic anemia) and gastrointestinal (nausea, vomiting, more rarely cholestatic jaundice) problems and allergic reactions. Sulfonylureas may also cause weight gain, and binding to plasma proteins can be potentiated by other drugs used concomitantly, causing hypoglycemia. This condition is the most worrisome adverse event observed and may be prolonged, which can have severe consequences in elderly patients, patients treated with multiple drugs and those with impaired renal function. Moreover, sulfonylureas stimulate appetite and can occasionally cause allergic rashes and bone marrow injury [45].

\subsubsection{Glimepiride}

The United States Food and Drug Administration (FDA) approved glimepiride in 1995 for the treatment of T2DM alone and in combination with metformin or insulin. It has prolonged 
action, lasting over 24 hours. Glimepiride has advantages with respect to its clinical and pharmacological profile, and it has also been shown to cause a lower incidence of severe hypoglycemia compared to other representatives of their class [48, 49].

Regarding hypoglycemia, the findings observed in some studies differ. In a systematic review and meta-analysis [50], we concluded that glimepiride caused more hypoglycemia compared to other sulfonylureas, and even more than other secretagogues. In other studies, the longacting sulfonylureas, such as chlorpropamide and glibenclamide, have been shown to be more likely to cause hypoglycemia [51-53]. In a UK survey, the rate of diagnosis of hypoglycemia was higher for glibenclamide compared to other representatives of the same class [51].

With regard to weight gain, in the United Kingdom Prospective Diabetes Study (UKPDS), the mean weight change after 10 years of follow up ranged from a minimum of $1.7 \mathrm{~kg}$ as a result of glibenclamide use to a maximum $2.6 \mathrm{~kg}$ with chlorpropamide use [19]. Glimepiride was claimed to be at least neutral with respect to body weight, and weight reduction has been observed by some authors [54, 55].

Sulfonylureas have different cross reactivities with cardiovascular ATP-dependent potassium channels. The closing of these channels by ischemic preconditioning can lead to cardiovascular mortality [56].

Several compounds increase the hypoglycemic effect of sulfonylureas, and several of these interactions are potentially important from a clinical standpoint. The non-steroidal antiinflammatory agents (including azapropazone, phenylbutazone and salicylates), coumarin, some uricosuric agents (e.g., sulfinpyrazone), alcohol, monoamine oxidase inhibitors, some antibacterials (including sulfonamides, and chloramphenicol trimethropim) and some antifungal agents (including miconazole and possibelmente, fluconazole) produce severe hypoglycemia when administered with sulfonylureas. The probable basis for these interactions is the competition for metabolizing enzymes, but interference in the plasma protein binding or excretion may also exert some effect. The agents that reduce the action of sulfonylureas include diuretics (thiazides and loop diuretics) and corticosteroids [45].

\subsection{Glinides}

Other insulin secretagogues drugs include repaglinide, nateglinide and mitiglinide. Derivatives of benzoic acid and the amino acid D-phenylalanine increase insulin secretion through an action similar to the sulfonylureas. Due to their rapid absorption, the action begins approximately 30 minutes after administration. These drugs have no interactions with other medication and are not contraindicated in pregnancy, lactation or in the presence of other pathologies

Repaglinide and nateglinide are insulin secretagogues with short action, with a half-life equivalent to 1 hour for the repaglinide and $1.5 \mathrm{~h}$ for nateglinide. Both of these secretagogues act by triggering an insulin peak during the postprandial period when administered before meals [57]. 


\subsubsection{Repaglinide}

Repaglinide ((S) - (+)-2-ethoxy-4-[2 - (3-methyl-1-[2 - (piperidin-1-yl) phenyl] Butylamino)-2oxoethyl] benzoic acid) was the first representative of the class of glinides, which differs from other classes of anti-hyperglycemic drugs due to its distinct molecular structure, mechanism of action and mechanism excretion [58].

This drug acts as a short-acting insulin secretagogue that targets the postprandial-released glucose. It significantly reduces the levels of plasma glucose in individuals with T2DM [59].

The mechanism of action of repaglinide involves blocking potassium efflux from pancreatic $\beta$ cells. This action depolarizes the cells by opening voltage-gated calcium channels. This process results in increased calcium influx into the B cells, which stimulates the exocytosis of insulin-containing secretory granules $[60,61]$. Regarding the pharmacokinetics, this drug has rapid absorption and rapid elimination, with a plasma half-life of up to 1 hour [61]. Repaglinide is primarily metabolized in the liver, and $90 \%$ of the drug is excreted through the gut and $8 \%$ through the urine [62]. Because the action of this drug is rapid and the time of effect is relatively short, it carries a low risk of hypoglycemia [63]. Repaglinide may be used as a monotherapy or in combination with other antidiabetic agents, such as metformin and glitazone [62].

\subsubsection{Nateglinide}

Nateglinide (3-phenyl-2-[(4-propan-2-ylcyclohexanecarbonyl) amino] propanoic acid) as well as repaglinide acts by inhibiting potassium channels that are sensitive to ATP, causing depolarization of the plasma membrane of the $\beta$ cell. This processes culminates in the influx of calcium ions into the cell and subsequent insulin secretion [64]. Nateglinide is thus an insulinotropic agent that is capable of restoring the physiological pattern of insulin secretion, which is not regulated in diabetic patients [65].

Potassium channels are known to be ATP-dependent in cardiac cells as well as in pancreatic $\beta$ cells. Thus, a reasonable degree of concern exists regarding the influence of therapeutic agents that act on these channels on heart function. Nateglinide has a high selectivity for pancreatic potassium channels and is more reliable in relation to repaglinide and glibenclamide with regard to the possible onset of cardiovascular events [65].

Nateglinide induces rapid and transient insulin secretion in a glucose-dependent manner. The response of potassium channels to nateglinide is remarkably lower during periods of euglycemia compared to periods when the glucose levels are high. Thus, the minimum total insulin exposure generated by this drug protects against hypoglycemia attacks, allowing the patient some flexibility regarding the intervals between meals [62].

The effects of nateglinide are specific to prandial insulin release, allowing for the observed reduction in glycated hemoglobin $(\mathrm{HbA} 1 \mathrm{c})$ without the risk of hypoglycemia between meals [65].

With regard to the pharmacokinetic properties, nateglinide is rapidly absorbed, and the peak plasma concentration is reached in 1 hour. The drug metabolism occurs in the liver, and approximately $10 \%$ is excreted unchanged via the kidneys [62]. 
As with repaglinide, nateglinide can be used as a monotherapy or in combination with other agents, such as metformin or glitazone. Both repaglinide and nateglinide have similar efficacy for reducing the fasting blood glucose levels and postprandial plasma glucose, and during early insulin secretion, they contribute to an improvement of insulin sensitivity and pancreatic $\beta$ cell function. However, repaglinide was shown to be more effective with regard to reducing $\mathrm{HbA1c}[65]$.

\subsubsection{Mitiglinide}

Mitiglinide is also an analogue of the meglitinides, and it has a mechanism of action that has been previously elucidated for other substances [60]. However, mitiglinide is not yet approved by the FDA for the treatment of T2DM.

\subsection{Treatment based on the effects of incretin hormones}

The use of incretin hormones in the treatment of type 2 diabetes is the subject of extensive investigations that have culminated in the development of new classes of drugs that have been recently approved for the treatment of the disease. The intensification of the action of incretin, especially GLP-1, is the basis of numerous new options for the metabolic control of T2DM.

Incretin mimetics, such as exenatide, as well as inhibitors of the enzyme DPP-4, such as sitagliptin and vildagliptin, have been developed. In general, these agents reduce the blood glucose levels to a level similar to that induced by other oral hypoglycemic agents with minimal risk of hypoglycemia. Furthermore, they have potential preventive effects and may promote disease regression. They may also have possible protective effects and promote growth in pancreatic $\beta$ cells.

\subsection{Antagonists of GLP-1R}

\subsubsection{Exenatide}

Exenatide is an exendin-4 GLP-1 mimetic with $~ 53 \%$ homology to endogenous GLP-1. It is currently approved for use as a monotherapy or in combination with metformin and/or sulphonylureas [66]. Exenatide is administered subcutaneously at a dose of 5 or $10 \mathrm{mg}$, twice a day. It binds to the GLP-1 receptor and has a longer lasting action because it presents greater resistance to the action of DPP-4 $[67,68]$.

In phase III studies, exenatide improved glycemic control in patients with type 2 diabetes for whom glycemic control was not achieved with metformin and / or sulfonylurea. These studies were double-blind, placebo-controlled, 30-week trials. The initial HA1c ranged from $8.2 \%$ to $8.7 \%$ and was reduced by approximately $1 \%$ when exenatide was compared with placebo. There was a mean weight loss of approximately $2 \mathrm{Kg}$. In the clinical trials that have been performed to date, the drop-out rate due to side effects of exenatide was less than $5 \%[69,70]$ The main adverse effects of exenatide are nausea, vomiting and diarrhea. Most episodes of nausea are mild to moderate and dose dependent, and they decrease in frequency with continued treatment [71]. 


\subsection{GLP-1 analogs}

\subsubsection{Liraglutide}

Liraglutide is a long-acting human GLP-1 analogue that shares 97\% amino acid sequence identity with human GLP-1 and is resistant to dipeptidyl peptidase-IV. Native GLP-1 has a short elimination half-life of 1-2 min, whereas liraglutide has a long half-life of approximately 13 hours and can be administered once a day [67, 72]. When administered once daily, liraglutide enables significantly superior glycemic control compared to that obtained with the administration of exenatide 2 times per day and is generally well tolerated [69].

Other GLP-1 analogs, taspoglutide and albiglutide are currently in phase III clinical trials. Albiglutide has a long half-life, allowing administration once per week.

\subsection{DPP-4 inhibitors}

\subsubsection{Vildagliptin}

Vildagliptin is a DPP-4 inhibitor that prolongs the activity of endogenous GLP-1. A study that compared vildagliptin with metformin showed a smaller decrease in HA1c after one year with vildagliptin, but fewer gastrointestinal side effects. Comparison of vildagliptin and pioglitazone showed similar reductions in HA1c [73].

Vildagliptin is used in several countries, but it has not been approved by the FDA because of the occurrence of elevated enzyme levels in patients taking higher doses of vildagliptin.

\subsubsection{Sitagliptin}

Sitagliptin is a DPP-4 inhibitor that was approved by the FDA in October 2006 to improve glycemic control in patients with type 2 diabetes. It is approved for use as a monotherapy or in combination with other oral hypoglycemic agents. It is administered at a dose of $100 \mathrm{mg}$ per day [74]. Treatment with this DPP-4 inhibitor is generally well tolerated. Sitagliptin monotherapy or in combination was shown to have a neutral effect on body weight. The incidence of hypoglycemia and gastrointestinal effects, such as abdominal pain, diarrhea, nausea and vomiting, was not significantly different between the groups treated with sitagliptin and placebo [75].

Sitagliptin displays a relatively low effectiveness in reducing HA1c and plasma glucose compared to other antidiabetic agents that have been utilized clinically for many years. Additionally, sitagliptin does not have beneficial effects beyond its effects on glycemic control, such as reduction in body weight [76].

Alogliptina and saxagliptin are still under investigation, and they have not been approved for use by the FDA. 


\subsection{Anti-obesity drugs}

Many clinical studies have reported the significant influence of excess body fat on metabolic disorders, such as T2DM. Notably, patients with T2DM who are significantly overweight (20\% to $40 \%$ ) have a higher mortality rate compared with patients with the same disease but who are within the proper range of weight. In many diabetic patients, a reduced calorie diet aimed at weight reduction alone is able to control blood glucose levels. However, for these individuals, body weight control is more complicated, both due to a mechanism of disease and for the treatment of disease. Thus, many doctors resort to drugs that are capable of inducing weight loss in patients with metabolic disorders [77].

In patients whose body mass index (BMI) is above $30 \mathrm{~kg} / \mathrm{m} 2$ or those with morbid conditions associated with overweight for whom non-pharmacological weight loss methods (diet, exercise) have failed, the use of antiobesity drugs is recommended [78]. These drugs may include medications with catecholaminergic action (amfepramone, femproporex, mazindol), serotonergic action (fluoxetine, sertraline) and mixed catecholaminergic and serotonergic action (such as sibutramine), which promote appetite control and induction of satiety. In addition to these drugs, tetrahydrolipstatin or orlistat may be used to inhibit lipase reducing intestinal fat absorption, allowing for a reduction of the dose of the hypoglycemic drug.

Amfepramone (diethylpropion), femproporex and mazindol are amphetamine derivatives. They work by stimulating the central nervous system, prompting the release of noradrenaline in the central and peripheral synapses. Through this mechanism, these drugs cause appetite suppression [79]. Amfepramone has been available in the market for weight loss since the 1960s [80]. It has been demonstrated in clinical trials with animals that mazindol stimulates the consumption of oxygen by increasing the stimulation of noradrenaline in the brown adipose tissue. Through this thermogenic effect, mazindol is capable of inducing weight loss [78].

The anorectic catecholaminergic drugs usually have good gastrointestinal absorption and are able to reach peak plasma levels within 2 hours of administration, and their metabolism is mainly hepatic. The catecholaminergic drugs have serious side effects, such as increased heart rate and blood pressure [78]. Generally, the use of amphetamine derivatives is accompanied by a remarkable pharmacodynamic tolerance, and their anorectic actions over the long term are not known [79].

Catecholaminergic and serotonergic medications have different effects on food intake. The former delay the start of ingestion, and the latter anticipate the completion of food intake [78].

Sibutramine acts by inhibition of noradrenaline and serotonin receptors, elevating the levels of these neurotransmitters in the hypothalamus and brainstem regions associated with energy homeostasis. This mechanism induces satiety and therefore the reduction of food intake. In addition to generating weight loss, sibutramine significantly reduces triglyceride and HDLcholesterol levels, its therapeutic action may be accompanied by some adverse effects, such as constipation, headache, and insomnia, which are usually mild [81]. However, cardiovascular events associated with the use of this substance, such as an increased heart rate and elevated blood pressure, are of concern [82, 83]. 
Orlistat is a reversible inhibitor of pancreatic and gastric lipase that induces weight loss by preventing the absorption of a significant amount of fat digested in the intestine [82]. It inactivates fat hydrolyzing, thereby reducing the absorption of calories by the patient [84]. The effect of orlistat on body weight reduction corresponds to benefits for other cardiometabolic parameters, such as blood pressure, waist circumference, and blood glucose. Among the most common adverse side effects of orlistat are diarrhea, flatulence, bloating and dyspepsia [81].

Although the weight loss caused by these drugs is generally not very significant, there is an improvement in insulin sensitivity and glycemic control in overweight patients who use these substances [84]. This effect emphasizes the importance of aid in the pharmacological management of obesity in patients with T2DM and other metabolic disorders; it is insufficient to only change the patient's lifestyle.

\subsection{Insulin}

The classification of insulin is based on the preparation. Namely, the duration of its action as short-acting, intermediate or basal insulin determines the classification. The latter two are the result of changes in crystalline insulin (short-acting). The addition of protamine and zinc result in Neutral Protamine Hagedorn (NPH) with intermediate and basal action, respectively.

The change in amino acid sequence allowed for the development of insulin analogues. The fast-acting insulin analogues lispro and aspart are available for clinical use and show similar pharmacokinetic and pharmacodynamic properties. The formulations glargine and detemir represent similar groups that may have either basal or long-term action (24 hours) [85].

\subsubsection{Short or ultra-rapid-acting insulin}

This group includes regular insulin and the analogues lispro, aspart and glulisine.

Regular insulin is usually administered by the subcutaneous route, often in combination with an intermediate-acting or long-duration insulin. Specific buffers are used to prevent crystallization due to its slow infusion. With this type of insulin, the monomers are presented in an associated hexamer form, which reduces the rate of absorption. Generally, regular insulin is indicated for the treatment of diabetic ketoacidosis, and it is also associated with human insulin intermediate-acting or basal analogs before meals [86]. This insulin must be given 30-45 minutes before meals to reduce the postprandial glycemia peak, and the activity lasts between 2 and 4 hours. However, patients tend to apply it at mealtime, which contributes to postprandial hyperglycemia and hypoglycemia in the period between meals because the regular insulin will peak at the time that the food has been metabolized. The first rapid-acting insulin analogue became available in 1996, and other rapid-acting analogs have been developed since. These analogues were produced by different modifications of the chemical structure of the human insulin protein, substituting various amino acids at different positions to shorten the onset and duration of action when compared to regular/ soluble insulin.

Insulin lispro is an analogue of human insulin developed using genetic engineering to reverse the amino acids proline and lysine in positions 28 and 29 of the beta chain, resulting in a 
sequence of Lys (B28) Pro (B29). This insulin in pharmaceutical preparations with phenol and zinc forms stable hexamers [86]. It has a lower tendency to self-aggregate at the site of subcutaneous injection, it is absorbed faster than regular human insulin, and it mimics the physiological insulin profile in response to a meal. Lispro begins to take effect within 5 - 15 minutes, and the duration of action is 1-2 hours [87]. During the use of these analogs, an additional dose is required in the afternoon to compensate for hyperglycemia that may result from an afternoon snack. There is evidence that compared with regular insulin, lispro insulin reduces the postprandial hyperglycemic peaks as well as the risk of hypoglycemia, especially at night [86].

In insulin aspart, a proline residue is replaced with a negatively charged aspartic acid at position 28 of the beta chain, producing electrical repulsion among the insulin molecules, which reduces their tendency for self-association. In vials or cartridges, the drug is present in the form of hexamers that rapidly dissociate into monomers and dimers in the subcutaneous tissue, ensuring rapid absorption. The pharmacokinetic profile includes the onset of action within 5 - 15 minutes and a duration of action of 1-2 hours [86].

The insulin glulisine is another insulin analogue with ultra-rapid action that is obtained by the exchange of asparagine for lysine at position 3 of the beta chain and lysine for glutamic acid at position 29 of the same chain. To date, there are few studies with glulisine, which seems to be similar to lispro and aspart with regard to its efficacy and the occurrence of hypoglycemic events. Due to its faster absorption, glulisine should be administered 5-10 minutes before a meal, ensuring greater flexibility for the patient and thus improving his/her quality of life. The short half-life reduces the need to eat food 2-3 hours after its administration, which is necessary with regular insulin, for which the greater half-life causes postprandial hypoglycemia. Although chemical structures of glulisine and insulin are different, no significant difference in time or duration of action was reported between them.

\subsubsection{Intermediate-acting insulin}

NPH insulin was introduced in 1946. It is a suspension of insulin in a complex with zinc and protamine in a phosphate buffer. Generally, a dose is given once a day before breakfast or twice daily. NPH has an absorption peak at approximately 4.6 hours after subcutaneous administration, followed by a steady decline in the level of plasma insulin [88]. This insulin can be mixed with regular insulin in the same syringe to increase patient compliance, especially in the case of children. However, the zinc present in the slow-acting insulin can prolong the effect of regular insulin [86].

The profiles of action of intermediate-acting insulin make them suitable for systems in which basal insulin is given one to three times daily. A rigid daily feeding programming is required, including a relatively fixed schedule for meals and snacks with consistent indices of carbohydrate meals / snacks. The major disadvantages of NPH are the wide variations in the daily timing and duration of the peaks within and between individuals, which, when compared to long-acting analogs, may result in non-optimal metabolic control and an increased risk for nocturnal hypoglycemia. The slow-acting insulin was used for many years as an intermediateacting insulin with a profile of action similar to that of NPH. 


\subsubsection{Basal insulin analogs (long-action insulin analogs)}

The insulins glargine and detemir belong to a group referred to as long-acting or basal insulin analogs [86].

Insulin detemir is produced by recombinant DNA technology, with expression in Saccharomyces cerevisiae followed by chemical modification [89]. A fatty acid (myristic acid) is attached to the lysine at position 29, which binds to circulating albumin, forming a complex that dissociates slowly, thus prolonging the duration of its action [79]. The insulin detemir is soluble at neutral $\mathrm{pH}$; however, it can be mixed with rapid analogs. Insulin detemir has shown potential benefits for body weight, with weight loss or decreased weight gain in adults, in children and in adolescents [90].

Insulin glargine is synthesized by introducing changes into the amino acid chain of human insulin, including a substitution of asparagine with glycine at position A21 and the addition of two arginines at position B30. These changes result in a standard single release from the injection site. That is, this analog precipitates in the subcutaneous tissue, allowing a gradual absorption into the bloodstream [91].

Basal insulin was developed to promote basal levels within 24 hours, and it may be administered once a day or at bedtime. When comparing conventional long-acting insulin with insulin glargine, it can be observed that insulin has a similar profile at a constant concentration without prominent peaks [92]. It has an onset of action between 1 and 2 hours, reaching the plateau of biological action between 4 and 6 hours with termination of the effect between 20 and 24 hours. Due to a slightly acidic $\mathrm{pH}$, glargine cannot be mixed with any other insulin in the same syringe; accordingly, some children complain of a burning sensation at the application site [86]. The timing of administration of glargine seems to have no impact on its efficacy for glycemic control, but the dose should be given at approximately the same time each day to maintain its efficacy as a free insulin peak action. If a dose is omitted, $50 \%$ of the daily insulin will be missed on that day.

\subsubsection{Inhalable insulin}

The benefits of injectable insulin are often limited, considering the difficulty of persuading patients to comply with the requirements for adequate treatment due to the need for multiple injections [93].

Aiming to alleviate this discomfort, the first US-approved inhaled insulin (Exubera ${ }^{\circledR}$ ) Pfizer / Nektar became available in January 2006. This product consisted of a dry powder formulation containing 1-3 g of human insulin administered via a single inhaler dose [94]. A polyethylene glycol inhalable dry powder that releases the equivalent of 3 to $8 \mathrm{UI}$ of short-acting insulin subcutaneously [95] was developed for this product. Although Exubera has demonstrated efficacy and a low risk of hypoglycemia, there was a poor acceptance by the prescriber and the patient, and in April 2008, the first clinical evidence that it may cause cancer emerged, with 6 cases diagnosed with lung cancer. There were also cases of primary lung malignancy in patients who had a history of smoking. Other important aspects include coughing, pulmonary function deterioration and an increase of anti-insulin antibodies [96]. 
AERx insulin was developed by Aradigm Corporation and Novo Nordisk. This system generates aerosol droplets from liquid insulin. The device guides the user to inhale reproducibly. It also offers the ability to download data related to the patient's insulin use, such as the frequency of inhalation, allowing for the monitoring of the treatment. Because of the experiences reported in the Exubera studies, their studies have been discontinued [97].

Technosphere Insulin (TI) is another system involving inhaled dry powder blends of recombinant human insulin (MannKind Corp.) using a MedTone ${ }^{\circledR}$ (Pharmaceutical Discovery Corp.) inhaler. This system is currently in phase II clinical research, and the partners have developed a placebo for inhalation, allowing for controlled and double-blind studies in patients with diabetes mellitus $[95,97]$.

\subsubsection{Insulin and cancer risk}

Studies report that chronic hyperinsulinemia is associated with the pathogenesis of colon cancer and also with breast, pancreas and endometrium cancer [98]. One possible mechanism that explains this relationship is that insulin resistance and hyperinsulinemia, which are characteristic of diabetic patients, or even increased levels of therapies based on endogenous insulin secretagogues or insulin, may increase the level of growth factor 1 of insulin, which plays an essential role in carcinogenesis [99]. Insulin receptors are present in (pre)-neoplastic cells, and insulin can stimulate growth. Furthermore, these cells may be susceptible to mechanisms that cause insulin resistance, such as subclinical chronic inflammation with increased TNF-alpha, which act as promoters of tumor growth. Obesity is associated with an increased risk of developing cancer due to factors such as the endocrine and metabolic effects and the consequent changes that they induce in the production of peptide and steroid hormones [98]. The results of a meta-analysis published in 2012 reported an increase of $28 \%$ in the risk of developing cancer in patients using insulin compared to non-users. Thus, there is growing that we are experiencing a worldwide epidemic of diabetes mellitus due to a large aging population and the increasing number of obese people who develop insulin resistance and hyperinsulinemia, which may further contribute to the number of patients with cancer [99].

The results of recently published meta-analyses indicate that some cancers develop most frequently in patients with diabetes, especially type 2 diabetes, although prostate cancer was shown to be less frequent in men with diabetes. Thus, preventive measures should be encouraged among the population to prevent these diseases [99].

\section{Conclusion}

Despite the clear benefits of achieving and maintaining glycemic goals and the availability of newer and potentially more effective drugs for the management of T2DM, the number of patients with poor glycemic control has not substantially decreased over the past 10 years.

The progressive nature of T2DM has been a significant challenge for achieving adequate glycemic control. The inability of classical oral antidiabetic agents to prevent the disease and 
maintain good metabolic control over the long term has motivated research on new physiological pathways involved in glucose homeostasis. The use of agents based on the effect of incretin hormones for the treatment of T2DM is quite promising. These treatments act through a mechanism that is distinct from that of drugs that have been commonly used in the treatment of this pathology. Furthermore, these agents reduce blood glucose levels to a level similar to other oral hypoglycemic agents with a minimal risk of hypoglycemia, they have few side effects, and they have no immediate need for dose titration.

However, although some of these agents have already been approved for the treatment of type 2 diabetes by regulatory authorities, phase IV studies and clinical experience are necessary to establish their actual benefits with greater certainty. The extra-glycemic effects, safety profiles and advantages and disadvantages compared to classically used oral antidiabetics remain to be fully characterized.

The increasing availability of numerous classes of medications has given clinicians and patients more therapeutic choices and perhaps an improved ability to achieve glycemic goals.

\section{Author details}

Roberto Pontarolo ${ }^{1^{*}}$, Andréia Cristina Conegero Sanches ${ }^{2}$, Astrid Wiens ${ }^{1}$, Helena Hiemisch Lobo Borba ${ }^{1}$, Luana Lenzi ${ }^{1}$ and Suelem Tavares da Silva Penteado ${ }^{1}$

*Address all correspondence to: pontarolo@ufpr.br

1 Department of Pharmacy - Federal University of Paraná, Curitiba, Paraná, Brazil

2 Department of Medical and Pharmaceutical Sciences - State University of West of Paraná, Cascavel, Paraná, Brazil

\section{References}

[1] World Health Organization. http://www.who.int/en/. (acessed 5 november 2012).

[2] George PM, Valabhji J, Dawood M, Henry JA. Screening for Type 2 diabetes in the accident and emergency department. Diabet Med 2005;22(12):1766-9.

[3] Chopra M, Galbraith S, Darnton-Hill I. A global response to a global problem: the epidemic of overnutrition. Bull World Health Organ 2002;80(12):952-8.

[4] Zimmet P, Shaw J, Alberti KG. Preventing Type 2 diabetes and the dysmetabolic syndrome in the real world: a realistic view. Diabet Med 2003;20(9):693-702. 
[5] U.S. Department of Health and Human Services. NDIC: National Diabetes Statistics. http://diabetesniddknihgov/dm/pubs/statistics/\#fast 2011. acessed in 12 november 2012.

[6] Boog M, Magrini V. Relato de experiência: reeducação alimentar por meio de abordagem interdisciplinar envolvendo as áreas de Nutrição e Saúde Mental. Revista da Sociedade de Cardiologia do Estado de São Paulo 1999;9(3):1-8.

[7] Hirsch I, Riddle M. Current therapies for diabetes. Endocr Clin North Am 1997 1997;26:3.

[8] Pan XR, Li GW, Hu YH, Wang JX, Yang WY, An ZX, et al. Effects of diet and exercise in preventing NIDDM in people with impaired glucose tolerance. The Da Qing IGT and Diabetes Study. Diabetes Care 1997;20(4):537-44.

[9] Hepler CD, Strand LM. Opportunities and responsibilities in pharmaceutical care. Am J Hosp Pharm 1990;47(3):533-43.

[10] American Diabetes Association. Economic consequences of diabetes mellitus in the U.S. in 1997. American Diabetes Association. Diabetes Care 1998;21(2):296-309.

[11] Donovan JL, Blake DR. Patient non-compliance: deviance or reasoned decision-making? Soc Sci Med 1992;34(5):507-13.

[12] Association AD. Standards of medical care in diabetes--2008. Diabetes Care 2008;31 Suppl 1:S12-54.

[13] Farmaco AId. L'uso dei farmaci in Italia - Rapporto nazionale anno 2007. 2007.

[14] Anderson J. Obesity. Br Med J 1972;1(5799):560-3.

[15] Cusi K, DeFronzo R. Metformin: a review of its metabolic effects. Diabetes Rev 1998;6:89-131.

[16] Santos RF, Nomizo R, Wajhenberg BL, Reaven GM, Azhar S. Changes in insulin receptor tyrosine kinase activity associated with metformin treatment of type 2 diabetes. Diabete Metab 1995;21(4):274-80.

[17] Johansen K. Efficacy of metformin in the treatment of NIDDM. Meta-analysis. Diabetes Care 1999;22(1):33-7.

[18] Araújo L, Britto MdS, Cruz T. Tratamento do Diabetes Mellitns do Tipo 2: Novas Opções. Arq Bras Endocrinol Metab 2000;44(6):509-18.

[19] Group U. Effect of intensive blood-glucose control with metformin on complications in overweight patients with type 2 diabetes (UKPDS 34). UK Prospective Diabetes Study (UKPDS) Group. Lancet 1998;352(9131):854-65.

[20] Chiasson JL, Josse RG, Hunt JA, Palmason C, Rodger NW, Ross SA, et al. The efficacy of acarbose in the treatment of patients with non-insulin-dependent diabetes mellitus. A multicenter controlled clinical trial. Ann Intern Med 1994;121(12):928-35. 
[21] DeFronzo R, Goodman A. The multicenter metformin study group: efficacy of metformin in NIDDM patients poorly controlled on diet done or diet plus sulfonylurea. N Engl J Med 1995;333:541-9.

[22] Inzucchi SE, Maggs DG, Spollett GR, Page SL, Rife FS, Walton V, et al. Efficacy and metabolic effects of metformin and troglitazone in type II diabetes mellitus. N Engl J Med 1998;338(13):867-72.

[23] Moses R, Slobodniuk R, Boyages S, Colagiuri S, Kidson W, Carter J, et al. Effect of repaglinide addition to metformin monotherapy on glycemic control in patients with type 2 diabetes. Diabetes Care 1999;22(1):119-24.

[24] Hirsch IB. Metformin added to insulin therapy in poorly controlled type 2 diabetes. Diabetes Care 1999;22(5):854.

[25] Turban S, Stretton C, Drouin O, Green CJ, Watson ML, Gray A, et al. Defining the contribution of AMP-activated protein kinase (AMPK) and protein kinase $C(\mathrm{PKC})$ in regulation of glucose uptake by metformin in skeletal muscle cells. J Biol Chem 2012;287(24):20088-99.

[26] Flier JS. Diabetes. The missing link with obesity? Nature 2001;409(6818):292-3.

[27] Kahn SE, Haffner SM, Heise MA, Herman WH, Holman RR, Jones NP, et al. Glycemic durability of rosiglitazone, metformin, or glyburide monotherapy. N Engl J Med 2006;355(23):2427-43.

[28] Olokoba AB, Obateru OA, Olokoba LB. Type 2 diabetes mellitus: a review of current trends. Oman Med J 2012;27(4):269-73.

[29] Silva A, Lazaretti-Castro M. Diabetes melito, tiazolidinedionas e fraturas: uma história inacabada. Arq Bras Endocrinol Metab 2010;54(4):345-51.

[30] Horton ES, Whitehouse F, Ghazzi MN, Venable TC, Whitcomb RW. Troglitazone in combination with sulfonylurea restores glycemic control in patients with type 2 diabetes. The Troglitazone Study Group. Diabetes Care 1998;21(9):1462-9.

[31] Iwamoto Y, Kosaka K, Kuzuya T, Akanuma Y, Shigeta Y, Kaneko T. Effect of combination therapy of troglitazone and sulphonylureas in patients with Type 2 diabetes who were poorly controlled by sulphonylurea therapy alone. Diabet Med 1996;13(4): 365-70.

[32] Day C. Thiazolidinediones: a new class of antidiabetic drugs. Diabet Med 1999;16(3): 179-92.

[33] Balfour JA, Plosker GL. Rosiglitazone. Drugs 1999;57(6):921-30; discussion 31-2.

[34] Grey A. Skeletal consequences of thiazolidinedione therapy. Osteoporos Int 2008;19(2):129-37. 
[35] van de Laar FA. Alpha-glucosidase inhibitors in the early treatment of type 2 diabetes. Vasc Health Risk Manag 2008;4(6):1189-95.

[36] Inzucchi SE, Bergenstal RM, Buse JB, Diamant M, Ferrannini E, Nauck M, et al. Management of hyperglycemia in type 2 diabetes: a patient-centered approach: position statement of the American Diabetes Association (ADA) and the European Association for the Study of Diabetes (EASD). Diabetes Care 2012;35(6):1364-79.

[37] Rosak C, Mertes G. Critical evaluation of the role of acarbose in the treatment of diabetes: patient considerations. Diabetes Metab Syndr Obes 2012;5:357-67.

[38] Coniff R, Krol A. Acarbose: a review of US clinical experience. Clin Ther 1997;19(1): 16-26; discussion 2-3.

[39] Lebovitz HE. alpha-Glucosidase inhibitors. Endocrinol Metab Clin North Am 1997;26(3):539-51.

[40] Braun D, Schönherr U, Mitzkat H. Efficacy of acarbose monotherapy in patients with type 2 diabetes: a double-blind study conducted in general practice. Endocrinol Metabol 1996;3:275-80.

[41] Coniff RF, Shapiro JA, Seaton TB, Hoogwerf BJ, Hunt JA. A double-blind placebocontrolled trial evaluating the safety and efficacy of acarbose for the treatment of patients with insulin-requiring type II diabetes. Diabetes Care 1995;18(7):928-32.

[42] van de Laar FA, Lucassen PL, Akkermans RP, van de Lisdonk EH, Rutten GE, van Weel C. Alpha-glucosidase inhibitors for patients with type 2 diabetes: results from a Cochrane systematic review and meta-analysis. Diabetes Care 2005;28(1):154-63.

[43] Holman RR. Long-term efficacy of sulfonylureas: a United Kingdom Prospective Diabetes Study perspective. Metabolism 2006;55(5 Suppl 1):S2-5.

[44] Wannmacher L. Antidiabéticos orais: comparação entre diferentes intervenções. Uso Racional de medicamentos: temas relacionados - Organização Pan-Americana da Saúde/Organização Mundial da Saúde 2005;2(11).

[45] Rang H, Dale M, Ritter J. Farmacologia. 4 ed. Rio de Janeiro: Guanabara Koogan; 2001.

[46] Akalin S, Berntorp K, Ceriello A, Das AK, Kilpatrick ES, Koblik T, et al. Intensive glucose therapy and clinical implications of recent data: a consensus statement from the Global Task Force on Glycaemic Control. Int J Clin Pract 2009;63(10):1421-5.

[47] Lee SJ, Eng C. Goals of glycemic control in frail older patients with diabetes. Jama 2011;305(13):1350-1.

[48] Basit A, Riaz M, Fawwad A. Glimepiride: evidence-based facts, trends, and observations. Vasc Health Risk Manag 2012;8:463-72. 
[49] Holstein A, Plaschke A, Egberts EH. Lower incidence of severe hypoglycaemia in patients with type 2 diabetes treated with glimepiride versus glibenclamide. Diabetes Metab Res Rev 2001;17(6):467-73.

[50] Gangji AS, Cukierman T, Gerstein HC, Goldsmith CH, Clase CM. A systematic review and meta-analysis of hypoglycemia and cardiovascular events: a comparison of glyburide with other secretagogues and with insulin. Diabetes Care 2007;30(2): 389-94.

[51] Ben-Ami H, Nagachandran P, Mendelson A, Edoute Y. Drug-induced hypoglycemic coma in 102 diabetic patients. Arch Intern Med 1999;159(3):281-4.

[52] Ferner RE, Neil HA. Sulphonylureas and hypoglycaemia. Br Med J (Clin Res Ed) 1988;296(6627):949-50.

[53] Shorr RI, Ray WA, Daugherty JR, Griffin MR. Individual sulfonylureas and serious hypoglycemia in older people. J Am Geriatr Soc 1996;44(7):751-5.

[54] Bugos C, Austin M, Atherton T, Viereck C. Long-term treatment of type 2 diabetes mellitus with glimepiride is weight neutral: a meta-analysis. Diabetes Res Clin Pract 2000;50(1):S47.

[55] Martin S, Kolb H, Beuth J, van Leendert R, Schneider B, Scherbaum WA. Change in patients' body weight after 12 months of treatment with glimepiride or glibenclamide in Type 2 diabetes: a multicentre retrospective cohort study. Diabetologia 2003;46(12):1611-7.

[56] Lazdunski M. Ion channel effects of antidiabetic sulfonylureas. Horm Metab Res 1996;28(9):488-95.

[57] Rosenstock J, Hassman DR, Madder RD, Brazinsky SA, Farrell J, Khutoryansky N, et al. Repaglinide versus nateglinide monotherapy: a randomized, multicenter study. Diabetes Care 2004;27(6):1265-70.

[58] Derosa G, Mugellini A, Ciccarelli L, Crescenzi G, Fogari R. Comparison between repaglinide and glimepiride in patients with type 2 diabetes mellitus: a one-year, randomized, double-blind assessment of metabolic parameters and cardiovascular risk factors. Clin Ther 2003;25(2):472-84.

[59] Stephens JW, Bodvarsdottir TB, Wareham K, Prior SL, Bracken RM, Lowe GD, et al. Effects of short-term therapy with glibenclamide and repaglinide on incretin hormones and oxidative damage associated with postprandial hyperglycaemia in people with type 2 diabetes mellitus. Diabetes Res Clin Pract 2011;94(2):199-206.

[60] Reimann F, Proks P, Ashcroft FM. Effects of mitiglinide (S 21403) on Kir6.2/SUR1, Kir6.2/SUR2A and Kir6.2/SUR2B types of ATP-sensitive potassium channel. Br J Pharmacol 2001;132(7):1542-8. 
[61] Wolffenbuttel BH, Nijst L, Sels JP, Menheere PP, Muller PG, Kruseman AC. Effects of a new oral hypoglycaemic agent, repaglinide, on metabolic control in sulphonylureatreated patients with NIDDM. Eur J Clin Pharmacol 1993;45(2):113-6.

[62] Dornhorst A. Insulinotropic meglitinide analogues. Lancet 2001;358(9294):1709-16.

[63] Papa G, Fedele V, Rizzo MR, Fioravanti M, Leotta C, Solerte SB, et al. Safety of type 2 diabetes treatment with repaglinide compared with glibenclamide in elderly people: A randomized, open-label, two-period, cross-over trial. Diabetes Care 2006;29(8): 1918-20.

[64] Keilson L, Mather S, Walter YH, Subramanian S, McLeod JF. Synergistic effects of nateglinide and meal administration on insulin secretion in patients with type 2 diabetes mellitus. J Clin Endocrinol Metab 2000;85(3):1081-6.

[65] Tentolouris N, Voulgari C, Katsilambros N. A review of nateglinide in the management of patients with type 2 diabetes. Vasc Health Risk Manag 2007;3(6):797-807.

[66] Fehse F, Trautmann M, Holst JJ, Halseth AE, Nanayakkara N, Nielsen LL, et al. Exenatide augments first- and second-phase insulin secretion in response to intravenous glucose in subjects with type 2 diabetes. J Clin Endocrinol Metab 2005;90(11):5991-7.

[67] Brown DX, Evans M. Choosing between GLP-1 Receptor Agonists and DPP-4 Inhibitors: A Pharmacological Perspective. J Nutr Metab 2012;2012:381713.

[68] Holst JJ, Deacon CF, Vilsboll T, Krarup T, Madsbad S. Glucagon-like peptide-1, glucose homeostasis and diabetes. Trends Mol Med 2008;14(4):161-8.

[69] Buse JB, Rosenstock J, Sesti G, Schmidt WE, Montanya E, Brett JH, et al. Liraglutide once a day versus exenatide twice a day for type 2 diabetes: a 26-week randomised, parallel-group, multinational, open-label trial (LEAD-6). Lancet 2009;374(9683):39-47.

[70] Kendall DM, Riddle MC, Rosenstock J, Zhuang D, Kim DD, Fineman MS, et al. Effects of exenatide (exendin-4) on glycemic control over 30 weeks in patients with type 2 diabetes treated with metformin and a sulfonylurea. Diabetes Care 2005;28(5): 1083-91.

[71] Iltz JL, Baker DE, Setter SM, Keith Campbell R. Exenatide: an incretin mimetic for the treatment of type 2 diabetes mellitus. Clin Ther 2006;28(5):652-65.

[72] Inoue K, Maeda N, Kashine S, Fujishima Y, Kozawa J, Hiuge-Shimizu A, et al. Shortterm effects of liraglutide on visceral fat adiposity, appetite, and food preference: a pilot study of obese Japanese patients with type 2 diabetes. Cardiovasc Diabetol 2012;10:109.

[73] Bloomgarden Z, Drexler A. What role will 'gliptins' play in glycemic control? Cleve Clin J Med 2008;75(4):305-10.

[74] Zerilli T, Pyon EY. Sitagliptin phosphate: a DPP-4 inhibitor for the treatment of type 2 diabetes mellitus. Clin Ther 2007;29(12):2614-34. 
[75] Gallwitz B. Review of sitagliptin phosphate: a novel treatment for type 2 diabetes. Vasc Health Risk Manag 2007;3(2):203-10.

[76] Nathan DM. Finding new treatments for diabetes--how many, how fast... how good? N Engl J Med 2007;356(5):437-40.

[77] Ye Z, Chen L, Yang Z, Li Q, Huang Y, He M, et al. Metabolic effects of fluoxetine in adults with type 2 diabetes mellitus: a meta-analysis of randomized placebo-controlled trials. PLoS One 2011;6(7):e21551.

[78] Mancini MC, Halpern A. Pharmacological treatment of obesity. Arq Bras Endocrinol Metabol 2006;50(2):377-89.

[79] Fuchs F, Wannmacher L, Ferreira M. Farmacologia clínica: fundamentos da terapêutica racional. Rio de Janeiro: Guanabara Koogan; 2004.

[80] Ioannides-Demos LL, Piccenna L, McNeil JJ. Pharmacotherapies for obesity: past, current, and future therapies. J Obes 2011;2011:179674.

[81] Kang JG, Park CY. Anti-Obesity Drugs: A Review about Their Effects and Safety. Diabetes Metab J 2012;36(1):13-25.

[82] Heal DJ, Gosden J, Smith SL. Regulatory challenges for new drugs to treat obesity and comorbid metabolic disorders. Br J Clin Pharmacol 2009;68(6):861-74.

[83] Sargent BJ, Moore NA. New central targets for the treatment of obesity. Br J Clin Pharmacol 2009;68(6):852-60.

[84] Li M, Cheung BM. Pharmacotherapy for obesity. Br J Clin Pharmacol 2009;68(6): 804-10.

[85] Maia F, Melo F. Substituição da insulina NPH por insulina glargina em uma coorte de pacientes diabéticos: estudo observacional. Arquivos Brasileiros de Endocrinologia \& Metabologia 2007;51(3):426-30.

[86] Pires A, Chacra A. A evolução da insulinoterapia no diabetes melito tipo 1. Endocrinol Metab 2008;52(2):268-78.

[87] Wajchenberg B, Forti A, Ferreira S, Oliveira O, Lopes C, Lerário A, et al. Menor incidência de hipoglicemia noturna com o uso de insulina lispro comparada à insulina humana regular no tratamento de pacientes com diabetes do tipo 1. Arq Bras Endocrinol Metab 2000;44(2):133-8.

[88] Home PD, Rosskamp R, Forjanic-Klapproth J, Dressler A. A randomized multicentre trial of insulin glargine compared with NPH insulin in people with type 1 diabetes. Diabetes Metab Res Rev 2005;21(6):545-53.

[89] Kurtzhals P. Pharmacology of insulin detemir. Endocrinol Metab Clin North Am 2007;36 Suppl 1:14-20. 
[90] Soran H, Younis N. Insulin detemir: a new basal insulin analogue. Diabetes Obes Metab 2006;8(1):26-30.

[91] Rollin G, Punales N. Utilização da insulina glargina em crianças menores de oito anos de idade. Arq Bras Endocrinol Metab 2009;53(6):721-25.

[92] Vazquez-Carrera M, Silvestre JS. Insulin analogues in the management of diabetes. Methods Find Exp Clin Pharmacol 2004;26(6):445-61.

[93] Bailey CJ, Barnett AH. Inhaled insulin: new formulation, new trial. Lancet 2010;375(9733):2199-201.

[94] Siekmeier R, Scheuch G. Inhaled insulin--does it become reality? J Physiol Pharmacol 2008;59 Suppl 6:81-113.

[95] Mastrandrea LD. Inhaled insulin: overview of a novel route of insulin administration. Vasc Health Risk Manag 2010;6:47-58.

[96] Arnolds S, Heise T. Inhaled insulin. Best Pract Res Clin Endocrinol Metab 2007;21(4): 555-71.

[97] Tibaldi JM. Evolution of insulin development: focus on key parameters. Adv Ther 2012;29(7):590-619.

[98] Carvalheira J, Saad M. Insulin resistance/hyperinsulinemia associated diseases not included in the metabolic syndrome. Arq bras endocrinol metab 2006;50(2):360-7.

[99] Janghorbani M, Dehghani M, Salehi-Marzijarani M. Systematic review and metaanalysis of insulin therapy and risk of cancer. Horm Cancer 2012;3(4):137-46. 
